\title{
Zusammenarbeit für eine bessere Nutzung von IT-Möglichkeiten
}

Gerhard Schillinga,

Peter Amherd ${ }^{b}$

a Präsident IPI, Vorstandsmitglied Hausärzte Schweiz

b Präsident VSFM, Leiter Marketing und Verkauf HCI Solutions AG
Korrespondenz:

Dr. med. Gerhard Schilling Chlini Schanz 42 CH-8260 Stein am Rhein Tel. 0527413626 Fax 0527413926
Die Vorstände beider Institutionen haben sich im Dezember 2012 zu Gesprächen getroffen. Zusammen hat man die gemeinsame Stossrichtung, die gemeinsamen Ziele und die gemeinsamen Aktivitäten entworfen und eine enge, aber unabhängige Zusammenarbeit initiiert. Dabei geht es einerseits um open Standards und die Migrierbarkeit der Daten, andrerseits um den Ausbau der elektronischen Krankengeschichten (eKG) mit hinterlegten Hilfstools. So sollen die vielfältigen Möglichkeiten von IT besser ausgeschöpft und damit Anreize und Mehrwert für die eKG, somit letztlich für die Ärztin und den Arzt, aber auch für die Patienten geschaffen werden.

Sowohl die Protagonisten des neugegründeten Institutes für Praxisinformatik (IPI) [1] wie auch die Vertreter aus dem Vorstand des Verbandes der Schweizerischen Fachhäuser für Medizinal-Informatik (VSFM) arbeiten seit Jahren daran, dass die Informatikmittel konsequenter in den Praxen in der Schweiz eingesetzt werden. Praktisch jede Arztpraxis hat heute Informatikmittel im Einsatz. Gut 80\% der Praxen setzen ihre Informatik-Infrastruktur aber nur reduziert ein - beispielsweise für das Abrechnen und das Berichtswesen. In der Grundversorgung arbeiten heute in der Schweiz lediglich knapp 25\% der Praxen mit der elektronischen Krankengeschichte. 75\% der Praxen haben sich noch nicht für eine Umstellung dieses zentralen Prozesses in der Praxis entschieden, oder teilweise lehnt man eine solche Veränderung die Entschlussfassung teilweise auch vor sich hergeschoben wird. Der Umstellungsprozess bei laufender Praxis ist zudem aufwendig. Die bisher fehlende Migrierbarkeit der Daten und die damit verbundene Abhängigkeit von ihrem Softwarelieferanten blockieren bei vielen Praxisbetreibern zusätzlich den Veränderungswunsch. Die Daten gehören dem dokumentierenden Arzt. Was aber, wenn man die Software wechseln will oder sich Praxen zusammenschliessen? Wie kann sichergestellt werden, dass die Daten vollumfänglich und verlässlich auch wieder in die neue Software integriert werden können? Diese Grundvoraussetzungen fehlten bisher.

\section{Die elektronische Dokumentation wird sich durchsetzen}

Dass sich die digitale Arbeitsweise auch beim Dokumentieren der medizinischen Inhalte durchsetzen wird, ist sowohl bei den Vertretern der Praxen/Ärzte wie auch auf der Seite der Industrievertreter aus der Branche der Softwarehäuser unbestritten. Die Frage ist: Wann wird der Grossteil der Praxen umstellen, aber auch, in welchem Umfang und vor allem mit welchen Inhalten soll oder muss dokumentiert werden? Was sind die rechtlichen und vertraglichen Konsequenzen einer Umstellung auf die digitale Dokumentation und Kommunikation? Die Ärzteschaft will verhindern, dass über den politischen Kanal oder über andere Interessengruppen aus dem

\section{Was sind die rechtlichen und vertraglichen Konsequenzen einer Umstellung auf digitale Dokumentation und Kommunikation?}

aus unterschiedlichen Gründen vorderhand kategorisch ab. Viele Entscheidungsträger in den Praxen sind verunsichert oder unschlüssig, es fehlt aber auch schlicht bis heute die notwendige Investitionssicherheit. Dies ist umso gravierender, als die ganze IT-Technologie tarifarisch in keiner Weise erfasst ist und der diesbezügliche finanzielle Aufwand beträchtlich ist.

Es mangelt aber auch an der nötigen Zeit, sich dieses Themas differenziert anzunehmen, weshalb
Gesundheitsmarkt praxisuntaugliche Ansätze in die Dokumentationsregeln einfliessen. Man will verständlicherweise selber bestimmen, was in welcher Form dokumentiert werden soll, und zu welchem Zweck welche Daten in die Krankengeschichte eingetragen werden. Die Ärzteschaft will die Qualität der Behandlungen weiter verbessern - auch mit Unterstützung der IT-Mittel. Die in den Krankengeschichten eingetragenen Daten bieten dafür die ideale Grundlage schon innerhalb der Praxis. Das 


\section{†े |P| InSTITUT FÜR \\ PRAXISINFORMATIK}

Am 22. Juni 2012 war es endlich so weit: Unter dem Namen «Institut für Praxisinformatik» (IPI) wurde in Luzern ein Verein gegründet.

\section{Das IPI ist eine gemeinsame Initiative von \\ - Hausärzte Schweiz (MFE) \\ - Konferenz der kantonalen Ärztegesellschaften (KKA) \\ - Institut für Hausarztmedizin Zürich (IHAMZ)}

\section{Der Zweck des IPI}

- Förderung der elektronischen Dokumentation (eKG) in den Arztpraxen und Sicherstellung der Praxistauglichkeit

- Drehscheibe und Verbindungsfunktion zwischen praktizierender Ärzteschaft, Software-Industrie und Politik

- Mithilfe bei der Grundlagenarbeit (open Standards, Migrierbarkeit, Grobarchitektur der eKG usw.)

- Dienstleistungen, Beratungen und Schulungen für die praktizierenden Ärzte

- Politisches Lobbying für die Praxisinformatik

Der Vorstand setzt sich aus folgenden Mitgliedern zusammen:

Dr. med. Gerhard Schilling, Präsident IPI und Vorstandsmitglied von Hausärzte Schweiz; Dr. med. Peter Wiedersheim, Vizepräsident IPI und Co-Präsident der Konferenz der Kantonalen Ärztegesellschaften; Dr. med. Marco Zoller, Kassier und Vertreter des Instituts für Hausarztmedizin der Universität Zürich (assoziiertes Mitglied); Dr. med. Marc-Henri Gauchat, Beisitzer und Co-Präsident der Konferenz der Kantonalen Ärztegesellschaften; Dr. med. Heinz Bhend, Exec. Master of ICT, fachlicher Leiter IPI und Vertreter von Hausärzte Schweiz; Dr. med. Urs Stoffel, ständiger Gast, ZV-FMH, Ressort eHealth 2

Weitere Informationen zum IPI finden Sie unter www.praxisinformatik.ch

\section{VSFM}

\section{Der VSFM - Verband Schweizerischer Fachhäuser}

für Medizinal-Informatik

Der Verband ist ein Verein im Sinne von Artikel 60ff. ZGB.

\section{Der Zweck des VSFM}

- die Interessen des Berufstandes zu wahren und zu fördern, insbesondere durch Stellungnahmen gegenüber der Öffentlichkeit und den Behörden, sowie durch Zusammenarbeit mit Institutionen, die sich ähnlichen Zielsetzungen widmen

- die Anwendung von Normen und Qualitätsstandards in der medizinischen Informatik zu fördern

- den fairen Wettbewerb in der Branche zu fördern

Die Zusammensetzung des Vorstandes und die Mitgliederliste finden Sie unter www.vsfm.info.
FIRE-Projekt [2] vorhanden (FIRE = family medicine icpc research using electronic medical records). Es gilt, diesen Weg für die Zukunft auszubauen und zu festigen. Die Softwarebranche kann hier mit dem Entwickeln von anwenderfreundlichen Tools dafür sorgen, dass die Daten schnell, einheitlich und in geeigneter und strukturierter Form während der Konsultationen erfasst werden können.

\section{IPI und VSFM: Zusammenlegen von praxis- nahem ärztlichem Wissen und technischer Kompetenz}

Damit aber die Softwarehäuser diese Entwicklungen überhaupt machen können, müssen sie Kenntnisse über das Wissen und die Bedürfnisse der Ärzteschaft haben und die Praxisabläufe kennen. Nicht jeder einzelne Arzt soll seine individuellen Ideen an die Softwarehäuser herantragen müssen. Das Institut für Praxisinformatik kann hier koordinierend wirken und mit dem Verband der Softwarehäuser zusammen Lösungsgrundlagen erarbeiten, die dann von allen Mitgliedfirmen des VSFM wiederum individuell in der Umsetzung genutzt werden können.

«Clinical Decision Support Systems» wird mittelfristig eines der zentralen IT-Themen sein. Geeignete Monitoringsysteme müssen entwickelt werden, damit am Arbeitsplatz in den Praxen echter Nutzen generiert werden kann. Die Softwarebranche kann dies nur tun, wenn sie über einheitliche Grundlagen verfügt, auf denen sie individuell die vorgegebene Datenerfassungsstruktur abbilden kann. Die Vorgaben für die fachlichen Grundlagen können nur von der Ärzteschaft her kommen. Die Zusammenarbeit des IPI mit dem VSFM ist die Basis, um genau diese Ziele gemeinsam umsetzen zu können.

\section{Viele Ärztinnen und Ärzte wollen in der Evaluation und bei der Umsetzung beraten und begleitet werden}

Auf dem Weg zur Entscheidungsfindung kann das IPI seine Dienste den Praxisbetreibern anbieten. Die Vertreter des Institutes können zum Nutzen der ganzen Praxis ihr eigenes Wissen einbringen. Sie arbeiten selber tagtäglich in ihren Praxen mit ICT-Systemen - und das schon seit vielen Jahren. Die Beraterinnen und Berater der Software-Branche haben teilweise sehr grosse Erfahrung und lösen ihre Aufgabe sehr gut. Oft fehlt ihnen aber der ganz enge Bezug zum Praxisalltag, was bei den Interessenten zu einer Verunsicherung führen kann. Die IPI-Vertreter können deshalb im Bedarfsfall in der Konzeptionsphase, in der Evaluations- und in der Einführungsphase ihre Dienste und ihr Know-how einbringen. Sie verstehen sich nicht als «Konkurrenten» der Berater aus den Softwarehäusern, sondern als neutrale, unterstützende Ergänzung - mit einem nachgewiesenen Praxisbezug. Die Geschäftsbeziehung zwischen den Softwareherstellern und ihren Kunden ist Sache dieser zwei Partner. Das IPI unterstützt
Potential und die Aussagekraft dieser Daten ist für die Forschung gewaltig. Die Absicht ist es daher, die Daten anonymisiert in noch grösserem Umfang zentral unter der Obhut der Ärzteschaft zu sammeln, sie auszuwerten und daraus Schlüsse zu ziehen - zum Nutzen der Praxisbetreiber in der ganzen Schweiz vor allem aber auch zum Nutzen der Patientinnen und Patienten. Bereits heute sind wertvolle Daten im 
lediglich mit Erfahrungen «aus der Praxis - für die Praxis». Es begleitet die Evaluierenden allenfalls beratend bei den entscheidenden Fragestellungen und kann in den Gesprächen ein gewisses «Coaching» übernehmen. Wo sinnvoll und vertretbar, kann das IPI - ergänzend zu den Abklärungs- und Umsetzungsschritten der Softwarehäuser - eine gewisse Systematik in die Projekte einbringen. Die im VSFM organisierten Softwarehäuser begrüssen eine solche Unterstützung und Begleitung.

\section{Das IPI unterstützt mit Erfahrungen «aus der Praxis für die Praxis».}

Kollektiven wie z. B. aus den Fachgesellschaften kam wenig Input. Umgekehrt hat sich die Softwarebranche über lange Zeit hinweg so verstanden, dass sie die medizinischen Anforderungen und Bedürfnisse wie auch die betriebswirtschaftlichen Belange genügend abdecken könne. Es braucht aber die fachliche Basis beider «Welten», um sowohl den Bedürfnissen der Ärzteschaft wie auch einer technisch einwandfreien Umsetzung der IT-Anforderungen gerecht zu werden. Genau hier wollen diese beiden Partner in der Zukunft eng zusammenarbeiten - und sich im Markt zum Nutzen der Praxisbetreiber ergänzen. Gemeinsam wurde eine klare Strategie mit verständlichen, gemeinsamen Zielen erarbeitet. Der Erfolg dieser Partnerschaft wird am Erreichen der gemeinsamen Ziele gemessen werden. Dazu braucht es eine gute und offene Kommunikation zwischen den Partnern. Die beiden Vorstände waren sich aber einig, dass man das Ziel einer alltagstauglichen Umstellung der Praxen auf die digitale Zukunft gemeinsam erreichen will. Bisher haben auch der Bund und eHealthSuisse dem Umstand einer praxistauglichen Umsetzung zu wenig Beachtung geschenkt. Die Kooperation der beiden Partner wird deshalb auch für die Politik und die eHealth-Strategie des Bundes positive Auswirkungen haben.

\section{Literatur}

1 Schilling G, Bhend H. IPI gegründet. PrimaryCare 2012; 12(13):237-8.

2 The FIRE project: a milestone for research in primary care in Switzerland. Chmiel C, Bhend H, Senn O, Zoller M, Rosemann T; Swiss Med Wkly. 2011;140:w13142. 